\title{
Study on Automatic Composition of Semantic Geospatial Web Service
}

\author{
Dacheng Wang ${ }^{1,2}$, Hongfeng $\mathrm{Wu}^{3}$, XiaodongYang ${ }^{1, *}$, \\ Wei Guo ${ }^{1}$, and Weihong Cui ${ }^{4}$ \\ ${ }^{1}$ National Engineering Research Center for Information Technology in \\ Agriculture, Beijing, 100097, China \\ ${ }^{2}$ Institute of Remote Sensing and GIS, Peking University, Beijing 100871, China \\ ${ }^{3}$ Institute of Scientific and Technological Informatics, Heilongiiang Academy of Land \\ Reclamation Sciences, 150038, Harbin, China \\ ${ }^{4}$ Institute of Remote Sensing Applications, Chinese Academy of Sciences, \\ Beijing, 100101, China
}

\begin{abstract}
We designed and implemented an integration framework of enterprise business information and multi-source heterogeneous geospatial information based on composition of semantic geospatial Web service. It can integrate business information and geospatial information seamlessly and effectively in enterprise workflow. An example of tobacco planting spatial analysis this framework was described to verify the practicability and feasibility of this framework.
\end{abstract}

Keywords: Semantic, Geographic information service, Automatic services composition, Enterprise geographic information integration.

\section{Introduction}

Since1990s, geographic information system(GIS) has came into enterprise applying stage gradually[1]. It has been applied widely in many fields such as land management, intelligent transportation, business analysis, and played a more and more important role. GIS promotes revolutionary changes in work patterns of these fields. With the accumulation of spatial information, data becomes more and more, and data sources become more and more complicated. Meanwhile, the association between GIS and general business data becomes stronger. But traditional GIS runs outside enterprise context data as an independent system which seriously limits the application of GIS in enterprise. Especially, the unique spatial decision support functions of GIS are often isolated in enterprise decision-making. In order to utilize the advantages of GIS in spatial decision support, how to realize the sharing and interoperability of heterogeneous geospatial information, and how to integrate them with enterprise context data seamlessly have been the current focus in geographic information research.

* Corresponding author. 
The sharing and integration of geographic information mainly goes through four stages [2]: file management stage, spatial database management stage, metadata information exchange stage and the current integration and interoperability of spatial information on the semantic level. The semantic Web can realize the sharing and thoroughly interaction of the resource on the internet on the semantic and knowledge level, especially the exact expression, sharing and interoperability of Web information and knowledge on the semantic level. The spatial data have the following characteristics: multiple sources, complicated structure, structure, mass data, implicit expression and complicated process.

So it is only the semantic Web service that can achieve the knowledge sharing and interoperability of spatial data on the syntax and semantic level.

Many foreign universities, research institutions and large companies have set up special project teams to promote the development of semantic Web technology. Among these project teams, W3C, DAML,SDK WSMO, LSDIS lab in Georgia University and the European Union IST research projects are representative in the semantic Web research. In 2002, the semantic Web technology was listed in the National 863 Program as a key support project. The NSF has also supported many research programs about the geospatial services on the semantic level. Which mainly includes 'Digital Government: Geospatial Knowledge Ontology' research programs developed by Findler and Malyankar, 'Spatial thinking and inference' research program hosted by Reginald G. Golledge, and 'IGERT: Geographic Information Science Training Scheme' hosted by David M. Mark and Barry Smith. Werner Kuhn, a researcher in the institute of geographic information science in Munster University, Germany, led the semantic interoperability laboratory and enhanced the availability of geospatial information through the implementation of semantic interoperability. It provided a semantic reference body for geospatial information. In addition, the application of geographic body in the transportation, environment and emergency systems was also studied. The CSISS Center at George Mason University studied the knowledge discovery of heterogeneous remote sensing images based on intelligent Web services under the support of NGA. The state key laboratory of remote sensing information engineering in Wuhan University carried out spatial information grid research based on geographic ontology.

\section{The Basis of Semantic Geospatial Services Technology}

\subsection{Web Services}

Web services are software systems which are used to support the network interoperability of the software interaction, whose interface can be described by the format (usually is WSDL) being automatically processed by machine. The other systems use SOAP message mechanism to communicate with it to the specified network services. Usually, they use the HTTP protocol and the combination of the XML serialization objects and other network standards to communicate message. 
Web services have the following characteristics [7]:

1) Good encapsulation. Web services are the objects which are deployed on the Web. So they have good encapsulation. The user can only need and see the function list provided for objects.

2) Loosely coupled. This feature stems from the object/component technology. As long as the call interfaces of Web services do not change, any changes in the implementation of Web services are all transparent. Even when the implementation platform of Web services migrates from J2EE to .NET or in the opposite direction, the user may know nothing about it.

3) Using standard protocol specifications. All public Web services protocols use the open standard protocols. In general, the majority of protocol norms will eventually use $\mathrm{W} 3 \mathrm{C}$ or OASIS as the publisher and maintenance side of the final version.

4) Highly integrated capability. Web services use simple and intelligible standard protocols as the norms of the component interface description and collaboration description. It shields the differences of different software platforms. So CORBA, DCOM or EJB can use a standard protocol for interaction operation to achieve highly integrated in the current environment.

\subsection{Semantic Web Services}

Tim Berners-Lee presented the concept and the architecture of semantic Web in 2000. The extensible markup language (XML), resource description framework (RDF) and ontology are at the core of this system [8]. The ontology as semantic layer helps human and machine to communicate clearly and supports exchange on the semantic level, not just on the syntax level. This layer describes the relation among various resources, and the ontology reveals more complex and richer semantic information of resource itself as well as among resources. Thus, it can separate structure from content of information and describe information formally to make the information online have computer-readable semantics. So the ontology plays an important role in the semantic Web. Meanwhile, it is the basis of solving the sharing and exchange of Web information on the semantic level.

The semantic Web services provide machine-readable description by adding semantic annotations for Web services. It can make automatic discovery, composition, invocation and monitoring services better in open, complex and chaotic network environment [9]. The Web Ontology Language for Service (OWL-S) is recommended by the World Wide Web Consortium (W3C) to describe the inputs and outputs of Web services, predefined conditions and the ontology layer in function and processing model. The OWL-S is based on the ontology Web language (OWL) which is a W3C recommended standard and presented by the Web-Ontology work group. The OWL is a markup language which can distribute and share data on the Internet by the ontology. Through OWL, ontology library can be created for any field so the instantiated ontology description can be provided to sites in any field. In addition, the OWL supports logical inference so it can ensure the logical relation and logical order of the ontology expression by automated inference. The $\mathrm{W} 3 \mathrm{C}$ does not give a specific definition of OWL-S, and just gives its main task to achieve [10]: 
1) Automatic Web services discovery: The automatic Web service discovery can automatically position and provide the specified functions. Meanwhile, it can meet the constraints of the client. Through the services utilizing the OWL-S semantic markup, it can standard out the information required by Web service using computer-readable semantic markup at Web services site. Thus, the enhanced search engine of service registry center or the ontology can mark up automatic positioning services by these semantic markups. In addition, service providers can also utilize the OWL-S to pre-advertising its services at registry center (that is middle agent). The service requester can find these services when they query the registry center. Therefore, the OWL-S supports the service properties of automatic service discovery and statement advertising of functions.

2) Automatic Web services invoking: The automatic Web service call just needs a declarative description of service. The application program or agent can automatically call the service and does not need to bind a particular service in pre-programmed way. The execution of Web service is similar to a series of remote call procedures. The OWL-S markup of Web services provides a declarative computer-readable API. It includes parameters and the semantics of the information returned after execution which is required during executing call. Software agents should be able to explain the inputs and the information returned required by calling service. The OWL-S combines with the domain ontology written in OWL and provides declarative API to automatically execute Web services.

3) Automatic Web services composition and interoperability: The automatic Web services composition and interoperability is to implement the automatic selection, composition and interoperability of Web services involved in a complex task after given abstract description of a task. Through the semantic markup of Web services, the information required by the selection and composition services can be encoded at the Web services site. Software can automatically process the description of task and complete the task. In order to support these functions, the OWL-S not only provides the prerequisite required by each atomic services and declarative definition of result information, but also provides a kind of descriptive services combination and data flow interaction language.

More and more companies begin to transform some their business processes into Web services which makes the company's business relatively independent and more conducive to maintenance. At the same time, the needs of enterprises become more and more complex. A single atomic Web services often can not meet the complicated and individualized requirements of enterprises. So the requirements of the Web services composition emerge in response to. the situation requires. The Web services composition aims to let the seamless integration of cross-enterprise processes and the life cycle of transaction have same type, and to be used for many Web services.

At present, business process execution language for Web services (BPEL4WS), Web services modeling ontology, OWL-S, Petri nets and pacification [11] are the commonly used methods in the Web services composition. The BPEL4WS is a static services composition mode. It executes the pre-binding workflow file using workflow processing engine. The advantage of this mode is the stability of the services composition, and its disadvantage is the poor adaptability. The WSMO, OWL-S, Petri 
nets and pacification all belong to the dynamic services composition mode. This mode dynamically searches and discovers services during the execution. Besides, it handles business processes using dynamic binding and composite services. It has good flexibility and adaptability. Among these methods, the OWL-S is the Web services composition standard recommended by the $\mathrm{W} 3 \mathrm{C}$. In the paper, this mode is also utilized.

\subsection{Geospatial Services}

At present, the geospatial services are the easiest way to access geographic data by using common network protocol. It is due to that geospatial services shield the differences between data formats and interfaces, and do not need to utilize traditional data distribution model [12]. The geospatial services have the potential to change the development, access and use of GIS, and make the sharing of geographic information data and functions easier [13]. Using the semantic Web technology, the geospatial services can be understood by other programs or software agents to achieve automatic composition of geographic services according to demand. It can provide more flexible and powerful functions. The ISO/TC211, OGC, INSPIRE and FGDC are all committed to research and develop the standards and norms of the geospatial services. The most important basis of the implementation of the semantic geospatial services is to establish a formal unified geographic ontology.

\section{Integration Framework Design of Semantic Geospatial Services}

The semantic geospatial services will have many advantages comparing with the existing geospatial services in many aspects. It will provide real-time and interactive geographic information services which also support spatial reasoning. The semantic geospatial services will support the seamless integration with general business management system. So the spatial information can be seamlessly managed as a part of a larger enterprise management system. This completely changes the island status of the geographic information in enterprise application.

The basis of achieving the semantic geospatial services integration is to establish the ontology library which meets the application requirements. According to the level of production, the ontology can be divided into three layers: Top-level ontology, domain ontology and application ontology. The top-level ontology usually describes the general concepts independent of any fields, such as event and object. The domain ontology is usually a concept in a specific field, such as the geographic information field and business logistics field. The application ontology is usually an application project oriented concept developed for a specific field and application.

Figure 1 shows the overall framework of the designed geospatial services integration in this paper. The basis of integration is to establish application ontology library 
according to the application requirements of project. This ontology library consists of agriculture, tobacco, logistics, commercial and much other ontology. Figure 2 shows the tobacco-growing geographic information application ontology object. The geospatial services and enterprise services achieve the sharing and interoperability of information using the semantics provided by this ontology library.

In figure 1, the service manager is implemented by a Web service. It responses to the inputs of user and parses the user's request into a service list. This service list contains the name and parameters of input and output of the required service, and receives the result data returned from the service composition manger. The service composition manger is also a Web service and implemented by Jena + OWL-S. Its main function is to combine service list sent by service manger into concept-level service composition chain. The service chain repository is used to save the executed service chain information which includes the composition state of service chain and the reference state of result data generated by executing service chain. The function of inference service and matching service is to change concept-level service composition chain into executive instance-level service composition chain through the semantic integration service. The semantic integration service provides the unified entrance for accessing geospatial services and enterprise application services.

A typical user request and response process is as follows:

1) User submits a request to the service manager by client;

2) Service manger parses the request into service list;

3) Service manger sends the service list to service composition manger. Service composition manger generates a concept-level service composition chain according to the input parameters.

4) Service composition manager looks for the instance of this service chain from instance service chain library. If it is found, directly return data reference address to the service composition manager. Service composition manager returns the address to service manager. Service manager executes this reference and return the result data to user.

5) If the instance of service chain can not be found, service composition manager calls the matching service and inference service. Through semantic integration services, the two services call the corresponding geospatial services and enterprise services to generate the instance of this service chain according to according to semantic information provided by the ontology library. After the instance service chain executes, the instance is returned to the service composition manager and is stored to service chain library. The service composition manager returns the address reference of result data generated by the execution of the instance service chain to service manager.

Before the execution of each atomic service of the instance service chain generated by inference service and matching service, the instance service chain library is checked first. If the instance of this atomic service is found, the result address reference of the instance is directly called. Otherwise, execute the specific service process after finding this instance service. 


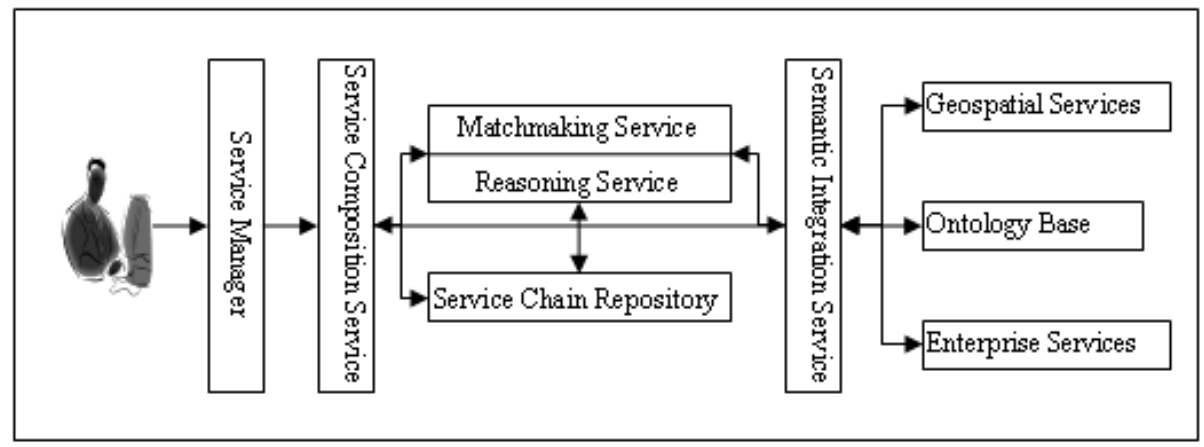

Fig. 1. Geospatial Service Integration Framework

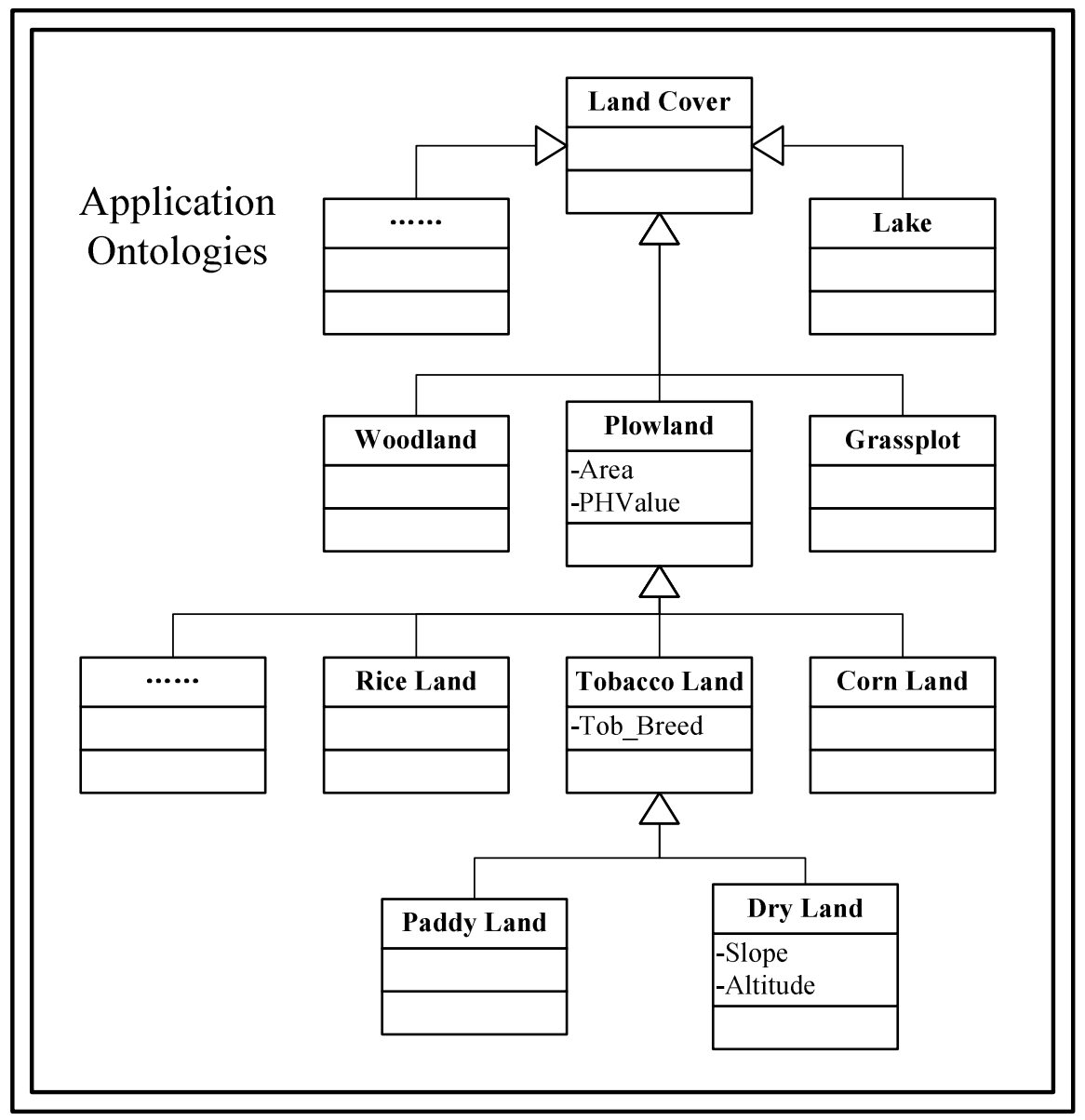

Fig. 2. Schema of Geospatial Ontologies in Tobacco Planting 


\section{$4 \quad$ An Example of Application}

Since China has joined the international "Framework Convention on Tobacco Control" in 2005, the State Tobacco Monopoly Bureau has begun to implement the strict "dual control" policy gradually, so the rational tobacco farming planning has become very important to tobacco manufactures. In the last two years, tobacco manufacturers in every district have begun to implement basic tobacco farming planning. Most of the basic data have been collected manually. The spatial data have been stored in paper maps and the attribute data have been stored in ordinary relational databases.

Due to the subjectivity and arbitrariness of the manual planning, companies now need to determine whether tobacco farm is suitable for growing tobacco. That is the 'whether a land suits to grow tobacco' problem.

Table 1. Conditions for Tobacco Planting

\begin{tabular}{|c|c|}
\hline Project & $\begin{array}{l}\text { Description of the appropriate } \\
\text { conditions }\end{array}$ \\
\hline Altitude & $1200-1900$ meters \\
\hline Slope & less than 15 degree \\
\hline Soil type & $\begin{array}{l}\text { Red soil, yellow soil, red and yellow } \\
\text { soil }\end{array}$ \\
\hline $\begin{array}{l}\text { The type of } \\
\text { land use }\end{array}$ & $\begin{array}{l}\text { Land, land on a field whose slope is } \\
\text { less than } 15 \text { degree }\end{array}$ \\
\hline Soil PH & $5.5-7$ \\
\hline Sunlight & $\begin{array}{l}\text { To large land, Sunlight duration is up } \\
\text { to } 500-700 \text { hours; sunshine rate is } \\
\text { greater than or equal to } 40 \% \text {; } \\
\text { Mining grilled period needs } \\
280-400 \text { hours; sunshine rate is } \\
\text { greater than or equal to } 30 \% \text { 。 }\end{array}$ \\
\hline Rainfall & $\begin{array}{l}\text { Lager land requires that the monthly } \\
\text { average rainfall is } 100-130 \mathrm{~mm}\end{array}$ \\
\hline Temperature & $\begin{array}{l}\text { The average daily temperature is } \\
\text { less than } 20 \text { centigrade from July to } \\
\text { September. The optimal temperature } \\
\text { is } 20-25 \text { centigrade. The } \\
\text { accumulated temperature is less than } \\
10 \text { centigrade in growth period }\end{array}$ \\
\hline
\end{tabular}


The temperature, humidity, sunshine, rainfall and other climatic conditions of tobacco growing have certain requirements. Meanwhile, tobacco growing needs appropriate soil type, land cover, slope, elevation and other geographic conditions. The climate data are often the statistical data of each administrative area issued by meteorological department. The geographic data often derives from the raster of vector map data issued by territory department and surveying and mapping department.

Whether a farm is suitable for the cultivation of tobacco depends not only on the elevation, slope, soil type, soil $\mathrm{PH}$, water conservancy facilities and other geographic data, but also on sunshine, rainfall, temperature and other statistical data. Table 1 shows the details.

The elevation and slope data are generated from the DEM data. The soil type distribution maps, $\mathrm{PH}$ value of soil distribution maps and land use distribution maps are obtained from the relevant departments, respectively. The sunshine, rainfall and temperature statistical data of each administrative area can be obtained from the meteorology bureau. Because the study area in this paper does not provide online data services, the data obtained in this paper are the original data files. In actual, the corresponding data services are established in the internal network. Table 2 shows the service list.

Table 2. Services used in the Application

\begin{tabular}{l|l}
\hline Content of service & Description of service \\
\hline MapServ & Vector map service \\
\hline FeatureServ & Feature service \\
\hline ImageServ & Image map service \\
\hline Overlay & Map overlay service \\
\hline Buffer & Buffer service \\
\hline SunlightComp & Sunlight statistical data service \\
\hline RainComp & Rainfall statistics service \\
\hline TemperatureComp & $\begin{array}{l}\text { Temperature statistical data } \\
\text { service }\end{array}$ \\
\hline
\end{tabular}

Assume that the user selects a piece of land from the client (usually a map page, also can be a data report) and then it is submitted to services manager for querying whether this land is suitable to grow tobacco. The process flow of the service request handled by the service manager is as follows: The existing service manager divides user request into atomic service list. The atomic service lists are combined into service list included two sub-service chain by the service composition manager. A sub-service chain is to determine whether the land conforms to the related spatial information conditions. Another sub-service chain is to determine whether the township where the land locates 
conforms to the related statistical conditions. Figure 3 shows the details of service chain process. Finally, the return value of the whole service(ie, appropriate level) is calculated according to the results returned by the two sub-service chains. The appropriate level can be calculated according to the following equation.

$$
\left\{\begin{array}{c}
Q_{a}=\sum k_{i}^{*} q_{i} \\
Q_{b}=\sum k_{j} * q_{j} \\
R=100 \% *\left(1-Q_{a}-Q_{b}\right)
\end{array}\right.
$$

Where $\mathrm{ki}, \mathrm{qi}, \mathrm{kj}, \mathrm{qj}$ represent the relevance degree and weights returned using the queried land as input parameters in a Web service, respectively; $\mathrm{R}$ is the appropriate level of growing tobacco in the queried land. Figure 4 shows the GUI of the system.

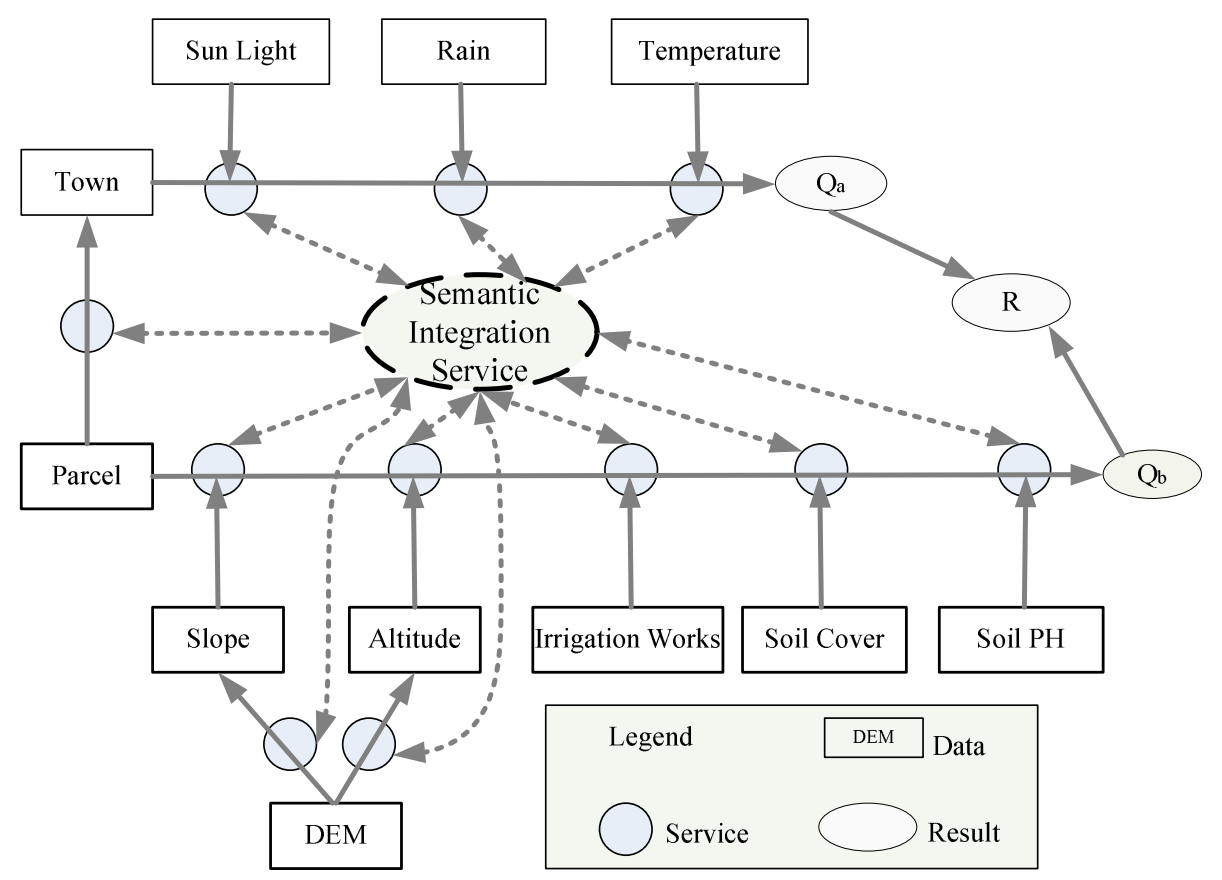

Fig. 3. Data Process Workflow of Business Instance 


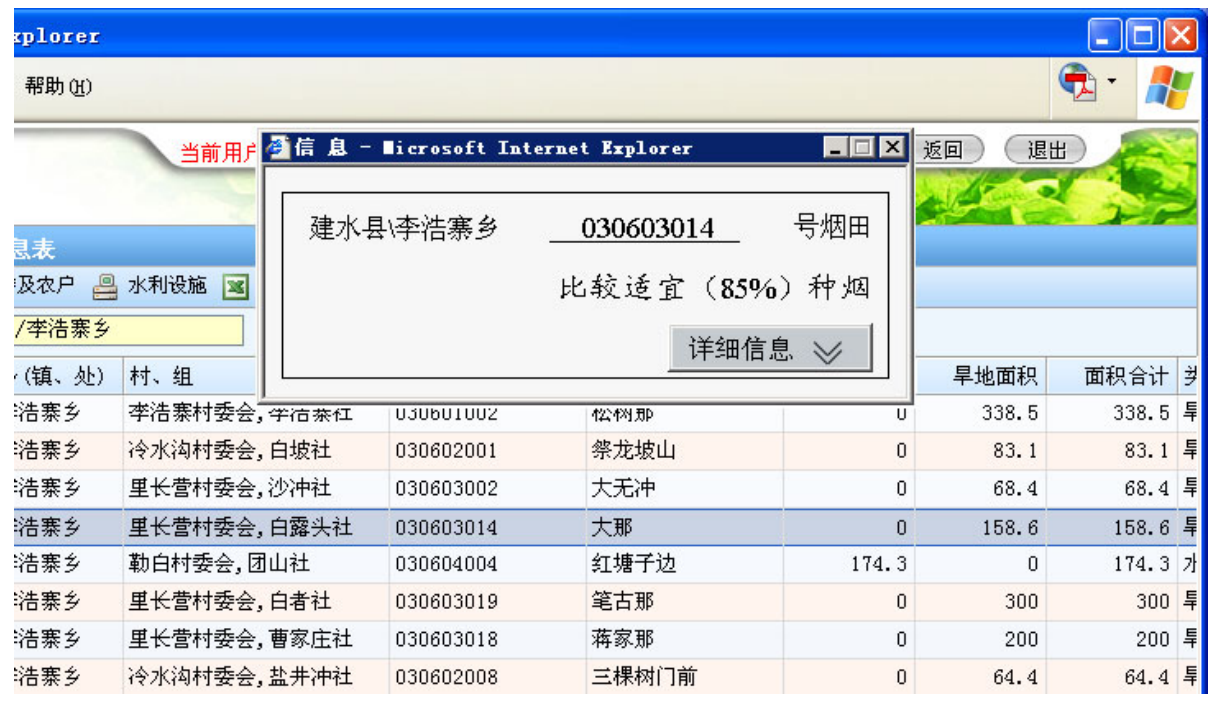

Fig. 3. A Snapshot of System UI

\section{Conclusions}

In this paper, the seamless integration and interoperability of the geospatial services in tobacco-growing enterprise application system is implemented under the framework of the geospatial services theory and technology. It promotes the application of the geospatial services in enterprise decision-making and meets the requirements of the enterprise geographic information application well.

The semantic geospatial services have wide prospect in the enterprise application. It is the development trend of the geospatial services enterprise integration. But it still has few mature applications at present. The further research and practice in this aspect will be conducted.

Acknowledgements. The work was supported by National Natural Science Foundation of China (Grant No.41101395 ) and Special Fund for Agro-scientific Research in the Public Interest (200903010).

\section{Reference}

[1] Song, G., Zhong, E., Liu, J., et al.: A Study on Seamless Integration of Multi-sources Spatial-data (SIMS). Progress in Geography (02) (2000)

[2] Wu, M.: Study on Ontology-driven Multi-Source Heterogeneous Spatio-temporal Data of Agricultural Geographical Information Taxonomy and Query. IRSA,CAS, 5 (2007) 
[3] Guide for Hi-tech Research and Development Program of China - Semantic Web Technology and Ontology [EB/OL] (May 8, 2002),

http: //www. 863.org.cn/863_105/applygide/applygide2/informati on2_app/200205080043.html

[4] Geospatial Knowledge Discovery via Intelligent Web Services[EB /OL] (October 10, 2007), http://www. laits.gmu. edu/geo/nga/index.html

[5] Li, D., Cui, W.: Geographic Ontology and SIMD. Acta Geodaetica Et Cartographica Sinica (02) (2006)

[6] Web Services Architecture [EB/OL] (February 11, 2004), http: / /www.w3 . org/TR/ws-arch/

[7] James, S.: Implementing Web Services with the WSTK [EB/OL]. IBM developerWorks (May 30, 2001)

[8] Yang, A.: Research on Geo Web Services Based on Ontology. Wuhan University (2005)

[9] Henriksson, R.: Semantic Web Service Composition [EB/OL] (November 26, 2006), http://www. cs.helsinki.fi/u/thruokol/opetus/2006/sose/papers /henriksson_sws-composition.pdf

[10] OWL-S: Semantic Markup for Web Services [EB /OL] (November 22, 2004), http: / /www.w3 .org/Submission/OWL-S/

[11] Charif, Y., Sabouret, N.: An Overview of Semantic Web Services Composition Approaches. Electronic Notes in Theoretical Computer Science 85(6) (2005)

[12] Breitman, K.K., Casanova, M.A., Truszkowski, W.: Semantic Web: Concepts, Technologies and Applications. Springer, London (2007)

[13] Tang, W., Selwood, J.: Connecting Our World: GIS Web Services. ESRI Press (2003)

[14] Rana, S., Sharma, J.: Frontiers of Geographic Information Technology. Springer, Berlin (2006)

[15] Yue, P., Di, L., Yang, W., et al.: Semantics-based automatic composition of geospatial Web service chains. Computers \& Geosciences 33, 649-665 (2007) 